\title{
Observations of GPS scintillation during an isolated auroral substorm
}

\author{
Keisuke Hosokawa ${ }^{1,2^{*}}$, Yuichi Otsuka ${ }^{3}$, Yasunobu Ogawa ${ }^{4}$ and Takuya Tsugawa ${ }^{5}$
}

\begin{abstract}
This paper reports simultaneous observations of ionospheric scintillation during an auroral substorm that were made using an all-sky full-color digital single-lens reflex (DSLR) camera (ASC) and a Global Positioning System (GPS) ionospheric scintillation and total electron content monitor (GISTM) in Tromsø (69.60 N, 19.20 E), Norway. On the night of November 19, 2009, a small substorm occurred in northern Scandinavia. The ASC captured its temporal evolution from the beginning of the growth phase to the end of the recovery phase. The amplitude scintillation, as monitored by the $\mathrm{S}_{4}$ index from the GISTM, did not increase in any substorm phase. By contrast, phase scintillation, as measured by the $\sigma_{\varphi}$ index, occurred when discrete auroral arcs appeared on the GPS signal path. In particular, the phase scintillation was significantly enhanced for a few minutes immediately after the onset of the expansion phase. During this period, bright and discrete auroral forms covered the entire sky, which implies that structured precipitation on the scale of a few kilometers to a few tens of kilometers dominated the electron density distribution in the E region. Such inhomogeneous ionization structures probably produced significant changes in the refractive index and eventually resulted in the enhancement of the phase scintillation.
\end{abstract}

Keywords: Auroral ionosphere; GPS scintillation; Auroral substorm; lonospheric density irregularities

\section{Background}

It is well known that small-scale electron density irregularities are frequently formed in the terrestrial ionosphere. At high latitudes, irregularities, which range in size from a few meters to a few tens of kilometers, often exhibit highly dynamic behavior when disturbed by solar wind and/or the magnetosphere (e.g., Tsunoda 1988). The consequent density perturbations sometimes induce significant fluctuations in the amplitude and phase of trans-ionospheric satellite transmission signals received on the ground, which are commonly called ionospheric amplitude and phase scintillations (Kintner et al. 2007). Amplitude scintillations are observed as random fluctuations of the signal strength, leading to periods of reduced signal strength for users of a global navigation satellite system (GNSS) such as the Global Positioning System (GPS). Phase scintillations are detected as high frequency fluctuations in the GNSS carrier phase, which may result

\footnotetext{
* Correspondence: keisuke.hosokawa@uec.ac.jp

'Department of Communication Engineering and Informatics, The University of Electro-Communications, 1-5-1 Chofugaoka, Chofu, Tokyo 182-8585, Japan ${ }^{2}$ Center for Space Science and Radio Engineering, The University of Electro-Communications, 1-5-1 Chofugaoka, Chofu, Tokyo 182-8585, Japan Full list of author information is available at the end of the article
}

in a loss of phase lock due to significant stress on the carrier phase tracking process (Skone 2001; Garner et al. 2011). Therefore, there is a strong requirement for modeling the trans-ionospheric satellite communication environment to reveal how ionospheric irregularities are formed during various disturbances in the high-latitude ionosphere.

One of the most dynamic ionospheric disturbances at high latitudes is the auroral substorm. These substorms are characterized by the sporadic precipitation of energetic electrons from the magnetotail that leads to explosive activations of aurora (Akasofu 1964). Aarons et al. (2000) first reported that fluctuations in a GPS signal phase correlate well with substorm-associated auroral disturbances as identified by the Polar Ultraviolet Imager instrument and local ground magnetometers. Recently, statistical maps of amplitude and phase scintillations at high latitudes were derived by Spogli et al. (2009) by using data from a regional network of a GPS scintillation monitoring system in northern Europe. They showed that, in the high-latitude ionosphere, phase scintillation is much more pronounced than amplitude scintillation and its occurrence frequency is clearly higher in the nighttime 
auroral region during the disturbance. This tendency has been confirmed by more recent statistical analyses of GPS ionospheric scintillation measurements (Li et al. 2010; Alfonsi et al. 2011; Tiwari et al. 2012). These studies suggest that the occurrence of phase scintillations is closely related to substorms. However, to date, investigations of the relationship between scintillation and substorm-related auroral disturbances have been primarily statistical; thus, the temporal evolution of the scintillation during a substorm has not yet been clarified.

According to auroral phenomenology (e.g., Akasofu 1964), substorms can be divided into growth, expansion, and recovery phases (Rostoker et al. 1980). When we observe a substorm using ground-based all-sky cameras, we normally see quiet east-west aligned discrete arcs during the growth phase. Such growth phase arcs tend to move slowly equatorward until the onset of the expansion phase. The expansion phase is characterized by a sudden increase in the brightness of one of the growth phase arcs and its subsequent poleward progression (Lyons et al. 2002). Following the poleward expansion, the aurora tends to become diffuse, indicating the start of the recovery phase. During the recovery phase, the aurora continues to become diffuse and patchy. Such patches often exhibit pulsation in their luminosity (pulsating aurora). As stated here, the type of auroral form varies drastically depending on the phase of the substorm; thus, the characteristics of ionospheric scintillation at auroral latitudes should be discussed in terms of the substorm phase. However, most previous auroral scintillation studies have investigated the response of GPS scintillation to the appearance of relatively quiet arcs at latitudes higher than the auroral region (i.e., in the polar cap region) (e.g., Kintner et al. 2007; Prikryl et al. 2013a; Kinrade et al. 2013). Recently, Prikryl et al. (2010, 2013b) introduced several intervals where moderate GPS scintillations were detected at auroral latitudes (Yellowknife and Fort Smith in Canada) in close association with auroral breakups observed by ground-based all-sky cameras. They clearly showed that there exists a close relationship between the activation of aurora and the occurrence of GPS scintillations. However, it is still unclear how the characteristics of GPS scintillations change during the three different substorm phases.

In this work, we investigated the temporal evolution of GPS scintillations during an auroral substorm on the night of November 19, 2009, through all-sky color digital camera observations of visible aurora together with ionospheric scintillation measurements using a GPS scintillation monitoring system in Tromsø, Norway. We observed a transient enhancement of the phase scintillation at the time of the onset of the substorm expansion phase. This result provides a new insight into how the characteristics of auroral scintillation can change depending on the phase of the substorm. By combining these simultaneous radio and optical observations, we discuss the temporal evolution of scintillations during substorms in terms of the direct production of irregularities caused by highly dynamic irregular auroral electron precipitation.

\section{Methods}

This paper presents simultaneous radio and optical observations of an auroral substorm made using an all-sky full-color digital single-lens reflex (DSLR) camera (ASC) and a GPS ionospheric scintillation and total electron content (TEC) monitor (GISTM) in Tromsø, Norway (69.60 N, 19.20 E). The ASC captures full-color auroral images at a rate of two frames per minute. The field-ofview (FOV) of the ASC shown in Figure 1 is mapped assuming the altitude of optical emission to be $110 \mathrm{~km}$, where concentric circles indicate the contours of the $30^{\circ}$, $60^{\circ}$, and $75^{\circ}$ zenith angles, respectively. We operated a dual frequency (L1: $1575.42 \mathrm{MHz}$ and L2: $1227.60 \mathrm{MHz}$ ) GPS receiver (GSV4004A) for monitoring scintillation and TEC. This measures the phase and amplitude of the

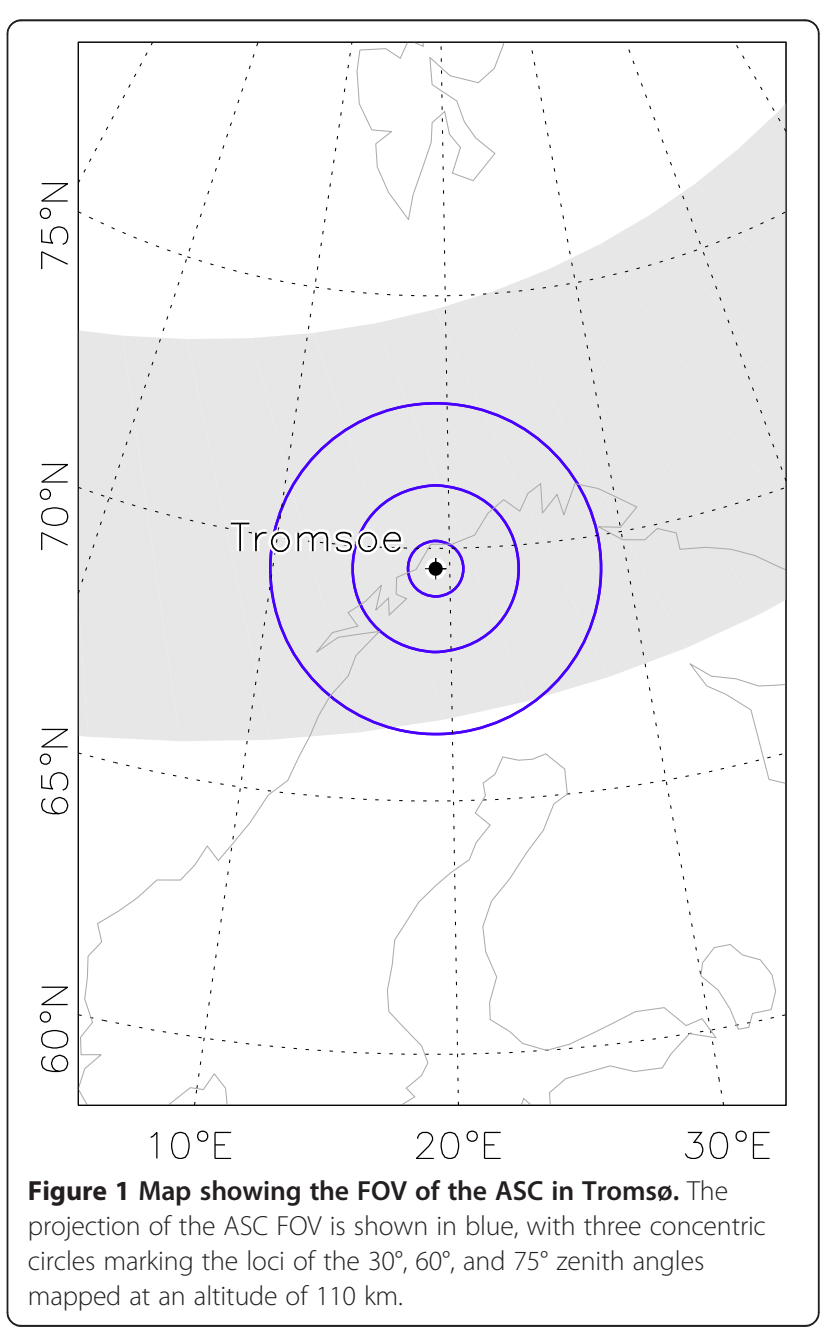


received signal at a temporal resolution of $50 \mathrm{~Hz}$ for each satellite tracked on L1 and calculates amplitude and phase scintillation indices in real time. Amplitude scintillation is monitored by estimating the $\mathrm{S}_{4}$ index, which is derived over $60 \mathrm{~s}$ intervals as the standard deviation of the signal intensity, normalized by its mean value. Phase scintillation monitoring is accomplished by deriving the standard deviation of the carrier phase, which is given in radians and referred to as $\sigma_{\phi}$. In the present analysis, $60 \mathrm{~s} \sigma_{\phi}$ values are used. The abovementioned GISTM and ASC were operated at the same location in Tromsø; thus, the data from the two systems can be compared directly.

\section{Results}

The interval presented is a $2 \mathrm{~h}$ period from 2100 to 2300 UT on November 19, 2009, during which a small substorm was observed by the ASC near midnight. Figure $2 \mathrm{~b}$ shows the ASC data in the form of a keogram, which is a time versus zenith angle plot of the optical intensity created from individual all-sky images. Here, we show a keogram along the magnetic south to north meridian. The onset of the substorm occurred at 2159 UT, which is marked by the dashed vertical line. During the growth phase, three discrete arcs were observed as indicated by the green, blue, and red arrows, respectively. These growth phase arcs showed some slight intensification; in particular, that denoted by the red arrow exhibited localized poleward expansion at around 2151 UT. However, none of them directly led to the onset on the expansion phase. About 15 min before the onset, another growth phase arc appeared well equatorward of the zenith as indicated by the white arrow. The expansion phase was initiated from this equatorwardmost arc. Such an initiation of auroral breakup from an equatorwardmost arc has been reported in a number of studies in the past (e.g., Lyons et al. 2002).

After the onset, multiple prominent auroral arcs dominated the FOV for about 5 min until 2204 UT. Subsequently, the luminosity decreased, and the form of the aurora became somewhat diffuse and patchy. At around 2218 UT, several bright discrete arcs appeared on the diffuse background near the zenith as denoted by the yellow arrow. After the disappearance of these arcs, the aurora became completely diffuse. Figure 2a shows the ground magnetic field X component (northward) obtained from a ground magnetometer of the International Monitor for Auroral Geomagnetic Effects (IMAGE) chain at Tromsø. The magnetogram was almost flat until the localized poleward expansion at 2151 UT. After that, the magnetic field data showed a negative excursion of approximately $300 \mathrm{nT}$, which is a typical signature of the expansion phase of auroral substorms. When the aurora became completely diffuse across the entire FOV at around
2220 UT, the magnetic field started increasing. This indicates that the aurora becoming fully diffuse marked the beginning of the recovery phase of the substorm.

Indices for monitoring amplitude and phase scintillations are shown in Figure 2d,e, respectively. During this 2-h period, signals from nine GPS satellites were received by the GISTM. The satellites were identified by the receiver by means of pseudo random noise (PRN) numbers ( 1 to 32 , indicated by color in Figure $2 \mathrm{~d}, \mathrm{e})$. In order to reduce the impact of tracking errors that are not directly related to ionospheric scintillation (e.g., multipath), only the indices computed from observations at elevation angles greater than $30^{\circ}$ are plotted. The level of amplitude scintillation as indexed by $\mathrm{S}_{4}$ was very low at less than 0.1 throughout most of the interval, even after the onset of the expansion phase. This is in good agreement with an earlier finding of Spogli et al. (2009), who statistically demonstrated that amplitude scintillation in GPS signals is generally very rare in the high-latitude ionosphere. The current observations further indicate that the stability of the amplitude of the GPS signal is almost unaffected even during the expansion phase of a substorm.

The level of phase scintillation as indexed by $\sigma_{\phi}$ was also low, at less than 0.1, during most of the growth phase. In contrast to the $S_{4}$ index, however, significant increases of $\sigma_{\phi}$ occurred immediately after the expansion phase onset for a few satellites including PRN13, PRN16, and PRN23. These large enhancements of $\sigma_{\phi}$ continued for approximately $5 \mathrm{~min}$ in the very early stage of the expansion phase. Although these enhancements were rather short-lived, $\sigma_{\phi}$ was still slightly higher than the quiet level (i.e., values during the growth phase) until around 2235 UT. In addition, there existed several transient increases of $\sigma_{\phi}$, for example, at around 2216 UT for PRN16 and at 2224 UT for PRN25. Thus, the level of phase scintillation was generally higher during the expansion and early recovery phases than that during the growth phase. After 2235 UT, the magnitude of $\sigma_{\phi}$ became comparable to the values during the growth phase, indicating that phase scintillation was no longer appreciable in the late recovery phase.

During intervals of auroral breakup on the nightside, regions of dense plasma are sometimes observed at the $\mathrm{F}$ region altitude. Such enhancements of $\mathrm{F}$ region plasma are manifestations of polar cap patches (e.g., Hosokawa et al. 2006), which are islands of dense $F$ region plasma transported from the dayside sunlit area across the central polar cap. Since polar cap patches are known to be one of the possible sources of scintillation in the high-latitude region (Prikryl et al. 2011), such structures might have produced the scintillations during the current interval. However, the time series of slant TEC shown in Figure 2c shows no remarkable large-scale enhancement; thus, the 


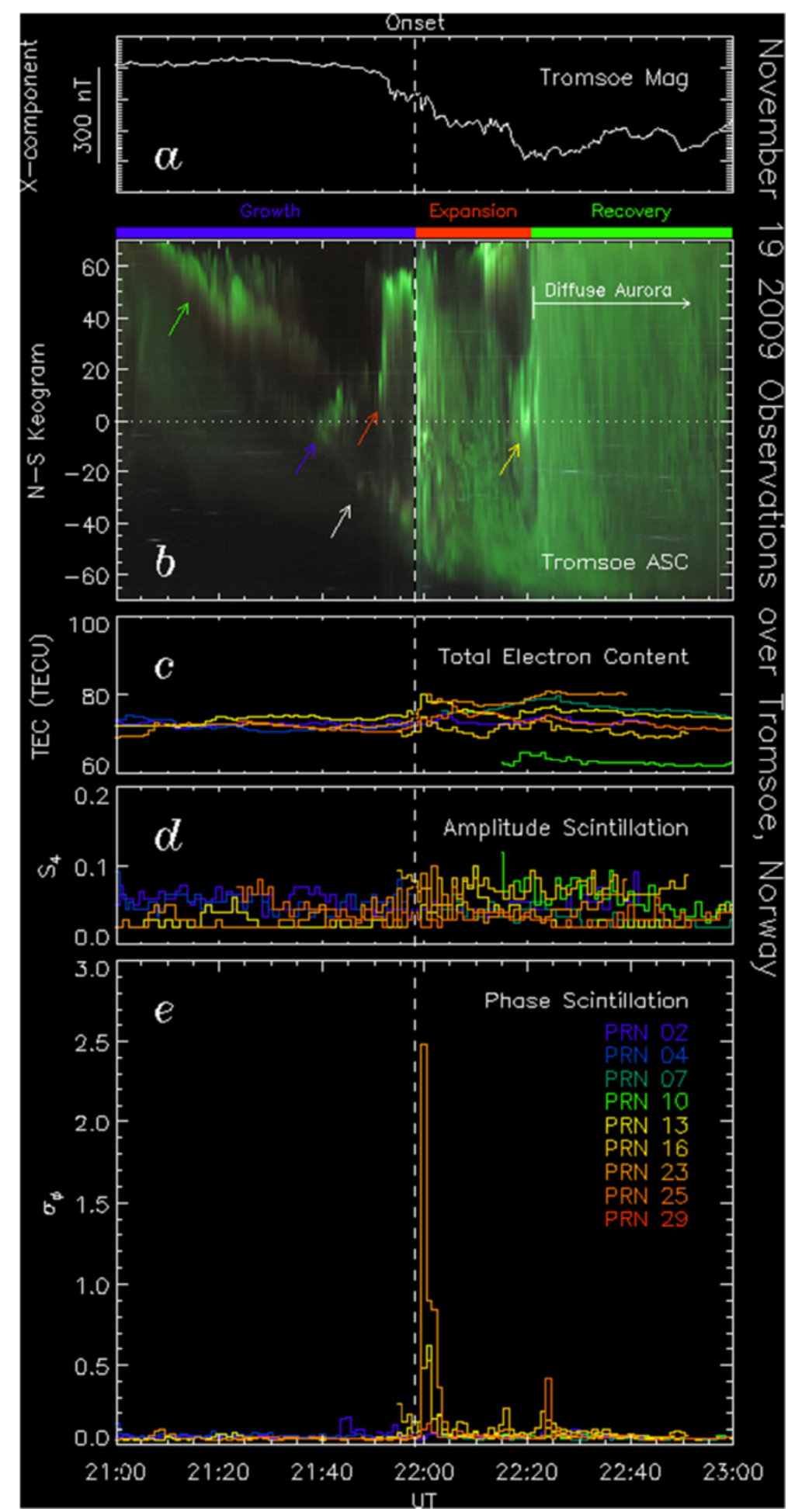

Figure 2 Summary of the measurements under study. (a) X-component magnetic field variation over Tromsø between 2100 to 2300 UT on November 19, 2009. (b) Keogram of the ASC along the south to north meridian, the vertical dashed line marking the onset of the substorm. (c) Slant TEC obtained from the GISTM observation. The color indicates the PRN number of the satellite. (d) $\mathrm{S}_{4}$ index estimated from the GISTM observation. The color indicates the PRN number of the satellite. (e) $\sigma_{\varphi}$ estimated from the GISTM observation. The color indicates the PRN number of the satellite. 
contribution of polar cap patches was almost negligible during the current substorm interval. Hence, the observed phase scintillations originated mainly from irregularities caused by auroral particle precipitation.

To examine the enhancements of $\sigma_{\phi}$ immediately after the onset of the expansion phase in more detail, we plot the $\sigma_{\phi}$ values computed for each satellite as a separate time series in Figure 3. Note that the scale of the vertical axis has been changed for PRN10, PRN23, and PRN29. The color indicates the PRN number of the satellite, and the grey portions of the lines mean that the elevation angle of the satellite was less than $30^{\circ}$ when the signal was received. Remarkable increases of $\sigma_{\phi}$ were detected immediately after the onset for PRN10, PRN13, PRN16, and PRN23, although the elevation angle of PRN10 was less than the threshold of $30^{\circ}$. These distinct enhancements of $\sigma_{\phi}$ continued for approximately 5 min until 2204 UT, suggesting that the impact of the substorm aurora on the phase of the GPS signal was extremely pronounced during the very early stages of the expansion phase. At this stage, however, we have not yet examined the 2D spatial relationship between the auroral signatures and the GPS satellite geometry. We revisit this point later in this section to confirm the

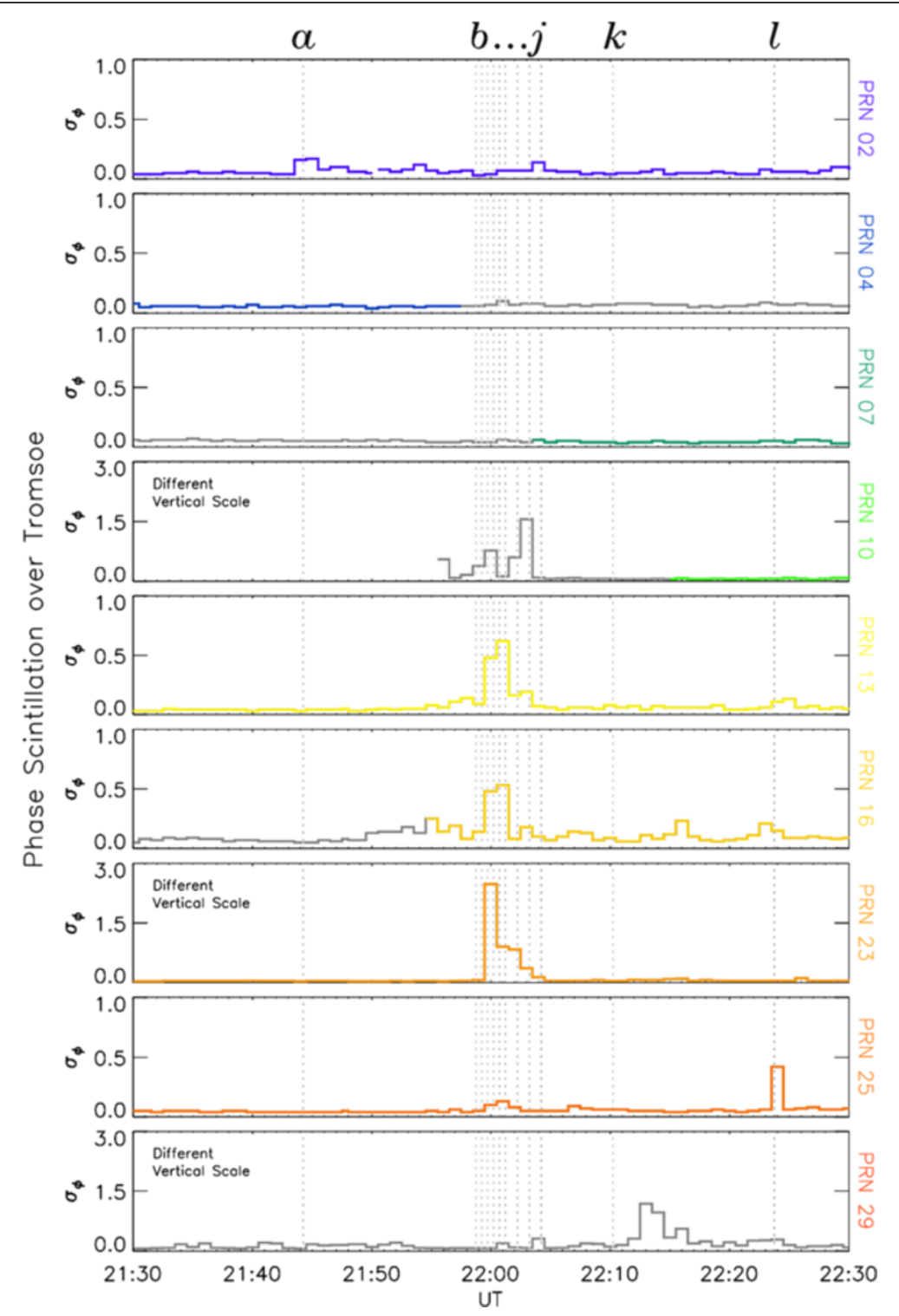

Figure $3 \sigma_{\varphi}$ values computed for each satellite shown as a separate time series. The color indicates the PRN number of the satellite, and the grey portions of the lines mean that the elevation angle of the satellite was less than $30^{\circ}$ when the signal was received. The scale of the vertical axis has been changed for PRN10, PRN23, and PRN29. The vertical dotted lines indicate the times at which the auroral images in Figure 4 were taken. 
impact of substorm aurora on the occurrence of phase scintillation.

Figure 3 demonstrates that the level of phase scintillation was still somewhat elevated after the onset. Such a tendency is more clearly seen in the data from PRN13, PRN16, and PRN25. For these satellites, $\sigma_{\phi}$ during the late expansion and early recovery phases was slightly higher than that during the growth phase. This again indicates that the substorm-related phase scintillation continued until the early recovery phase, although it was not as large as that immediately after the onset. As mentioned above, some appreciable enhancements of $\sigma_{\phi}$ can be identified before and well after the onset, for instance, peaks at around 2144 UT for PRN02 and 2224 UT for PRN25. Interestingly, these satellites did not observe significant enhancements of $\sigma_{\phi}$ near the onset. This implies that the occurrence of scintillation is highly dependent on the satellite's location. Specifically, the position of the satellite relative to the auroral features could be one of the factors determining the occurrence of the phase scintillation.

In Figure 3, it is shown that remarkable enhancements of $\sigma_{\phi}$ were identified over a period of a few minutes immediately after the onset. However, to confirm the direct impact of the substorm aurora on the occurrence of phase scintillation, we need to clarify the spatial relationship between the satellites and the aurora arcs. In Figure 4, the ASC data are shown in an all-sky image format together with the positions of the satellites. The optical intensity is plotted as a function of elevation and azimuthal angle without any assumptions on the height of the emission layer. Since the position of the GPS satellites is also a function of the elevation and azimuthal angles, we can compare the two data sets without any assumptions on the mapping height. The color of the point corresponds to the PRN number of the satellite, and grey points indicate that the elevation angle of the satellite was less than the threshold of $30^{\circ}$ when the signal was received. The times at which these images were taken are marked by the vertical dotted lines in Figure 3. At 2144:14 UT (Figure 4a), a thin growth phase arc was observed near the zenith. All satellites were located outside the arc except PRN02, which was the only satellite to experience an increase in $\sigma_{\phi}$ at this time. The next two images (Figure 4b,c) were obtained immediately prior to the onset. The aurora was somewhat intensified, but most of the satellites were located in darker areas adjacent to the brighter arcs; thus, $\sigma_{\phi}$ did not increase significantly at this stage. This again confirms that the occurrence of scintillation is highly dependent on the location of the satellites relative to the auroral arcs.

The image at 2159:44 UT (Figure 4d) was taken exactly at the onset of the expansion phase, after which the entire FOV was filled with extremely bright auroral arcs until 2205:14 UT (Figure 4k). During the poleward expansion, almost all of the satellites were located within the aurora. PRN13, PRN16, and PRN23 were deeply embedded in the prominent auroral forms in particular, which led to the large increase in $\sigma_{\phi}$ for these satellites. In contrast, PRN02 and PRN25 were located somewhat away from the bright aurora, which could, in turn, explain the weak or lack of increase in $\sigma_{\phi}$ for these satellites. After 2205:14 UT (Figure 4k), the brightness decreased gradually and the aurora became relatively diffuse; this is a typical temporal evolution of auroral substorms. At the time of the last image (Figure 4l), the data from PRN25 showed a distinct increase in $\sigma_{\phi}$. The ASC data show that a discrete aurora appeared above the diffuse background as a thin arc roughly aligned north to south. Since PRN25 was located very close to this arc, the observed increase in $\sigma_{\phi}$ was directly caused by the transient appearance of the north-south arc.

The abovementioned relationship between the occurrence of phase scintillation and auroral activities can be seen more clearly in the animation accompanying the electronic version of this article (Additional file 1). The movie shows ASC images captured at a rate of one frame every $30 \mathrm{~s}$ from the early stage of the growth phase (2030 UT) to the end of the recovery phase (2330 UT), together with the geometry of the GPS satellites. The color of the point indicates the PRN number of the satellite, and grey points indicate that the elevation angle of the satellite was less than the threshold of $30^{\circ}$ when the signal was received. In addition, the size of the points is proportional to the level of the phase scintillation as indexed by $\sigma_{\phi}$. A larger point indicates that enhanced phase scintillation was observed for that satellite. The movie clearly demonstrates that the phase scintillation became significantly enhanced immediately after the onset of the expansion phase and that the enhancement was quite intermittent.

\section{Discussion}

Throughout the substorm interval studied in this paper, no appreciable increase in amplitude scintillations, which are indexed by $\mathrm{S}_{4}$, was detected. This was the case even when the whole FOV of the ASC was filled with discrete auroral arcs moving dynamically. On the other hand, phase scintillations were observed more frequently, although their occurrence was highly dependent on the phase of the substorm, i.e., the form of the aurora. During the growth phase, the level of phase scintillation was mostly low. However, weak scintillations were identified occasionally when discrete auroral arcs appeared in the GPS signal path. This suggests that the relative position of the GPS satellite to the discrete arc is an important factor in the occurrence of phase scintillations, which is consistent with past observations (e.g., Prikryl et al. 2013a, 2013b). Immediately after 


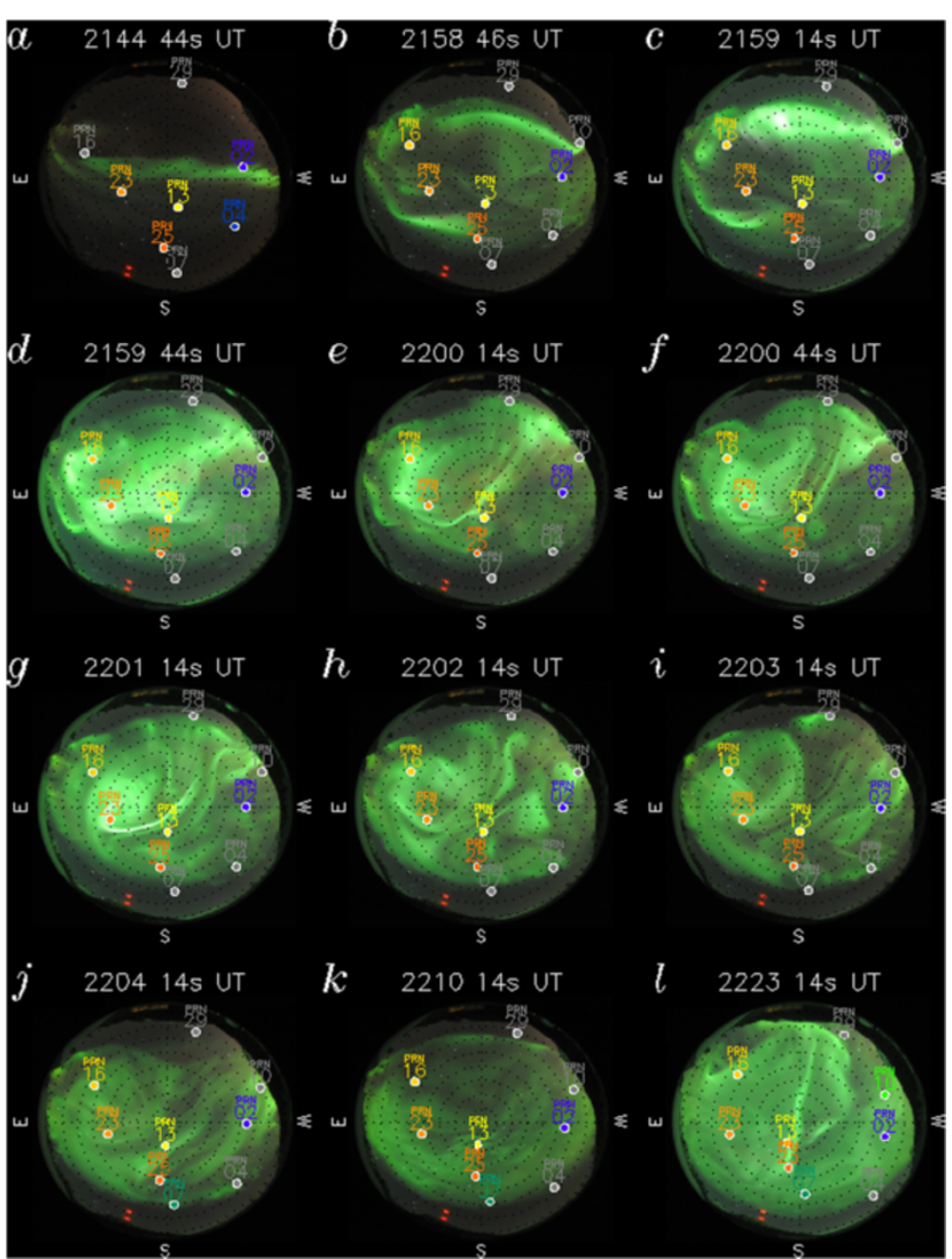

Figure 4 Selected ASC all-sky images together with the location of the satellites. (a to I) Selected ASC all-sky images together with the location of the satellites. The color of the point gives the PRN number of the satellite, and the grey points mean that the elevation angle of the satellite was less than the threshold of $30^{\circ}$ when the signal was received. The times at which these images were taken are marked by the vertical dotted lines in Figure 3.

the onset of the expansion phase, moderate/intense phase scintillations $\left(\sigma_{\phi}>0.5 \mathrm{rad}\right)$ were detected in almost half of the GPS signals tracked by the receiver. This was due to the entire FOV of the receiver being filled with bright auroral features moving very dynamically. However, this drastic increase in the phase scintillation continued for just $5 \mathrm{~min}$ following the onset of the expansion phase; the level of phase scintillation decreased back to the original level as the auroral form became more diffuse. During the recovery phase, in which diffuse aurora covered the entire FOV, considerable increases in the phase scintillation were not apparent. This indicates that the level of phase scintillation is generally very low during the recovery phase. Even in the recovery phase, however, weak phase scintillations sometimes appeared in a localized area where discrete auroral arcs appeared on the diffuse background structure. This again implies that an existence of discrete aurora in the GPS signal path is necessary for the occurrence of phase scintillation during substorm intervals. That is, phase scintillations are produced when the signals from GPS satellites propagate through regions of electron density perturbed by discrete aurora.

Ionospheric scintillations occur when the signal from a satellite propagates through a disturbed region of the ionosphere. The reason for this may be due to diffraction when the signals are modulated during their propagation through plasma irregularities in the ionosphere, the refractive index of which is continually subject to small variations (Kintner et al. 2007). An electromagnetic wave impinging such a medium with a spatially uniform phase will exit the medium with a spatially irregular phase. After 
propagating to a receiver, the signals are diffracted and a fluctuation in the wave amplitude is detected on the ground. Such diffractive scintillations are produced by ionospheric irregularities, the scale of which is comparable to the first Fresnel radius $r_{F}=\sqrt{2 \lambda r}$, where $\lambda$ is the incident signal wavelength and $r$ is the distance from the irregularities to the receiver (Kintner et al. 2007). For the GPS L1 signal, $r_{F}$ is approximately $500 \mathrm{~m}$, assuming the altitude of the irregularities to be $350 \mathrm{~km}$ and a signal path elevation of $90^{\circ}$. The amplitude scintillation is known to be due to diffraction; thus, the scale of the irregularities producing amplitude scintillation must be around $500 \mathrm{~m}$ for GPS L1 signals. The cause of scintillation may be refraction when the signal propagates through a medium of disturbed phase velocity (e.g., Kintner et al. 2007). In this case, propagating plane waves remain plane waves, but their phase can change due to the integrated phase shift across the disturbed medium. Phase scintillations are typically produced by variations in the refractive index due to ionospheric irregularities of larger scale (a few to a few tens of kilometers) moving across the signal path (Kintner et al. 2007). Hence, it is likely that the phase scintillations during the current interval were produced through the refractive process caused by large-scale density irregularities, which are probably structured ionizations associated with rapidly moving discrete auroral arcs. We derived the phase scintillation index every minute using 3,000 data points sampled at a temporal resolution of $50 \mathrm{~Hz}$, which means that the enhancement of $\sigma_{\phi}$ is caused by fluctuations in the phase whose temporal scale ranges from 0.04 to $60 \mathrm{~s}$. If we assume the speed of the horizontal motion of the auroral arcs to be approximately $1 \mathrm{~km} \mathrm{~s}^{-1}$, which is often the case at the onset of the expansion phase (e.g., Hosokawa et al. 2013), 0.04 to $60 \mathrm{~s}$ phase fluctuations correspond to electron density structures of $40 \mathrm{~m}$ to $60 \mathrm{~km}$ in size. These scales are in good agreement with that of the discrete auroral arcs typically present during the substorm interval (e.g., Partamies et al. 2010).

In previous studies, such refractive phase scintillations were observed in the form of phase fluctuations, which are defined as the rate of change of the TEC (e.g., Pi et al. 1997). Aarons (1997) and Aarons et al. (2000) investigated the phase fluctuations detected by 12 GPS receivers in a high-latitude region and demonstrated that the phase fluctuations were largest in the auroral oval near magnetic midnight. Coker et al. (1995) also indicated that $\mathrm{E}$ region ionization associated with auroral electron precipitation can potentially cause fluctuations in the TEC, inducing refractive scintillations. More recently, Kintner et al. (2002) employed observations from a ground-based all-sky camera in the late morning sector to demonstrate that the ionization at $\mathrm{E}$ region altitude associated with individual discrete auroral arcs could produce refractive scintillations or simply rapid changes in the TEC (i.e., phase fluctuations). During the current interval, the dynamic behavior of the breakup aurora in the first $5 \mathrm{~min}$ after the onset likely produced rapidly moving ionization structures at $\mathrm{E}$ region altitudes. The fast motion of these ionization structures then resulted in rapid changes in the refractive index of the GPS signal path, which contributed to the extreme enhancement of the phase scintillation immediately after the onset. The current observations also showed that phase scintillations were not apparent during the growth and recovery phases of the substorm in which the motion of the aurora tends to be slower. Considering that the aurora was still well spatially structured even during the growth and recovery phase, it is possible that the rapid motion of the ionization structure is the primary cause of the phase scintillation during the early stage of the expansion phase.

\section{Conclusions}

In this study, we investigated the effects of auroral substorms on ionospheric scintillations in the GPS L1 band. Amplitude scintillation did not increase during any substorm phase. In contrast, phase scintillation occurred when discrete auroral arcs appeared in the signal path. In particular, phase scintillation significantly increased for a few minutes immediately after the onset of the expansion phase during which very bright discrete aurora forms covered the entire sky and moved very dynamically. It was thus likely that an ionization structure of a few to a few tens of kilometers in size moved across the GPS signal path, leading to significant changes in the refractive index and resulting in the enhancement of the phase scintillation. In order to confirm this hypothesis, we plan to extend this study through the use of $50 \mathrm{~Hz}$ GPS phase data, electron density measurements made by the European Incoherent SCATer (EISCAT) radar and high-time-resolution optical data in the future. In addition, to confirm and evaluate the impact of the substorm aurora on the phase scintillation, it is necessary to carry out a statistical analysis using a number of GISTM measurements during substorms.

\section{Additional file}

Additional file 1: Relationship between the occurrence of phase scintillation and auroral activities during 3-h interval from 2030 to 2330 UT on November 19, 2009.

\section{Abbreviations}

ASC: All-sky Camera; FOV: field-of-view; GISTM: GPS ionospheric scintillation and TEC monitor; GPS: Global Positioning System.

\section{Competing interests}

The authors declare that they have no competing interests. 


\section{Authors' contributions}

$\mathrm{KH}$ proposed the topic and conceived and designed the study. YUO developed the GPS scintillation monitoring system installed in Tromsø. YUO and YAO installed the GPS scintillation monitoring system in Troms $\varnothing$, and YUO calibrated the raw data. YAO maintains all-sky digital camera system in Troms $\varnothing$. $\Pi$ collaborated with the corresponding author in the production of the manuscript. All authors read and approved the final manuscript.

\section{Acknowledgements}

This work was supported by JSPS KAKENHI Grant Number 23403010. We thank the institutes who maintain the IMAGE Magnetometer Array. We are also indebted to H. Miyaoka for installing the ASC in Tromsø in 2004.

\section{Author details}

${ }^{1}$ Department of Communication Engineering and Informatics, The University of Electro-Communications, 1-5-1 Chofugaoka, Chofu, Tokyo 182-8585, Japan. ${ }^{2}$ Center for Space Science and Radio Engineering, The University of Electro-Communications, 1-5-1 Chofugaoka, Chofu, Tokyo 182-8585, Japan. ${ }^{3}$ Solar-Terrestrial Environment Laboratory, Nagoya University, Furo-cho, Chikusa-ku, Nagoya, Aichi 464-8601, Japan. ${ }^{4}$ National Institute of Polar Research, 10-3 Midoricho, Tachikawa, Tokyo 190-8518, Japan. ${ }^{5}$ National Institute of Information and Communications Technology, 4-2-1

Nukui-Kitamachi, Koganei, Tokyo 184-8795, Japan.

Received: 27 December 2013 Accepted: 14 July 2014

Published: 4 August 2014

\section{References}

Aarons J (1997) Global positioning system phase fluctuations at auroral latitudes. J Geophys Res 102:17219-17231

Aarons J, Lin B, Mendillo M, Liou K, Codrescu M (2000) Global positioning system phase fluctuations and ultraviolet images from the polar satellite. J Geophys Res 105:5201-5213

Akasofu S-I (1964) The development of the auroral substorm. Planet Space Sci 12:273-282

Alfonsi L, Spogli L, De Franceschi G, Romano V, Aquino M, Dodson A, Mitchell CN (2011) Bipolar climatology of GPS ionospheric scintillation at solar minimum. Radio Sci 46, doi:10.1029/2010RS004571

Coker C, Hunsucker R, Lott G (1995) Detection of auroral activity using GPS satellites. Geophys Res Lett 22:3259-3262

Garner TW, Harris RB, York JA, Herbster CS, Minter CF III, Hampton DL (2011) An auroral scintillation observation using precise, collocated GPS receivers. Radio Sci 46, doi:10.1029/2010RS004412

Hosokawa K, Shiokawa K, Otsuka Y, Nakajima A, Ogawa T, Kelly JD (2006) Estimating drift velocity of polar cap patches with all-sky airglow imager at Resolute Bay, Canada. Geophys Res Lett 33, doi:10.1029/2006GL026916

Hosokawa K, Milan SE, Lester M, Kadokura A, Sato N, Bjornsson G (2013) Large flow shears around auroral beads at substorm onset. Geophys Res Lett 40, doi:10.1002/grl.50958

Kinrade J, Mitchell CN, Smith ND, Ebihara Y, Weatherwax AT, Bust GS (2013) GPS phase scintillation associated with optical auroral emissions: first statistical results from the geographic South Pole. J Geophys Res 118:2490-2502, doi:10.1002/jgra.50214

Kintner PM, Kil H, Deehr C, Schuck P (2002) Simultaneous total electron content and all-sky camera measurements of an auroral arc. J Geophys Res 107, doi:10.1029/2001JA000110

Kintner PM, Ledvina BM, de Paula ER (2007) GPS and ionospheric scintillations. Space Weather 5, doi:10.1029/2006SW000260

Li G, Ning B, Ren Z, Hu L (2010) Statistics of GPS ionospheric scintillation and irregularities over polar regions at solar minimum. GPS Solut 14, doi:10.1007/ s10291-009-0156-X

Lyons LR, Voronkov IO, Donovan EF, Zesta E (2002) Relation of substorm breakup arc to other growth-phase auroral arcs. J Geophys Res 107, doi:10.1029/ 2002JA009317

Partamies N, Syrjasuo M, Donovan E, Connors M, Charrois D, Knudsen D, Kryzanowsky Z (2010) Observations of the auroral width spectrum at kilometer-scale size. Ann Geophys 28:711-718

Pi X, Mannucci AJ, Lindqwister UJ, Ho CM (1997) Monitoring of global ionospheric irregularities using the Worldwide GPS Network. Geophys Res Lett 24:2283-2286
Prikryl P, Jayachandran PT, Mushini SC, Pokhotelov D, MacDougall JW, Donovan E, Spanswick E, St Maurice J-P (2010) GPS TEC scintillation and cycle slips observed at high latitudes during solar minimum. Ann Geophys 28:1307-1316

Prikryl P, Jayachandran PT, Mushini SC, Chadwick R (2011) Climatology of GPS phase scintillation and HF radar backscatter for the high-latitude ionosphere under solar minimum conditions. Ann Geophys 29:377-392

Prikryl P, Ghoddousi-Fard R, Kunduri BSR, Thomas EG, Coster AJ, Jayachandran PT, Spanswick E, Danskin DW (2013a) GPS phase scintillation and proxy indices observed at high latitudes during a moderate geomagnetic storm. Ann Geophys 31:805-816, doi:10.5194/angeo-31-805-2013

Prikryl P, Zhang Y, Ebihara Y, Ghoddousi-Fard R, Jayachandran PT, Kinrade J, Mitchell CN, Weatherwax AT, Bust G, Cilliers PJ, Spogli L, Alfonsi L, De Franceschi G, Romano V, Ning B, Li G, Jarvis MJ, Danskin DW, Spanswick E, Donovan E, Terkildsen M (2013b) An interhemispheric comparison of GPS phase scintillation with auroral emission observed at South Pole and from DMSP satellite. Ann Geophys 56, doi:10.4401/ag-6227

Rostoker G, Akasofu S-I, Foster J, Greenwald RA, Kamide Y, Kawasaki K, Lui ATY, McPherron RL, Russell CT (1980) Magnetospheric substorms: definition and signatures. J Geophys Res 85:1663-1668

Skone SH (2001) The impact of magnetic storms on GPS receiver performance. J Geophys Res 75:457-468

Spogli L, Alfonsi L, De Franceschi G, Romano V, Aquino MHO, Dodson A (2009) Climatology of GPS ionospheric scintillations over high and mid-latitude European regions. Ann Geophys 27:3429-3437

Tiwari S, Jain A, Sarkar S, Jain S, Gwal AK (2012) lonospheric irregularities at Antarctic using GPS measurements. J Earth Syst Sci 121:345-353

Tsunoda RT (1988) High-latitude F region irregularities: a review and synthesis, Rev Geophys 26:719-760

doi:10.1186/2197-4284-1-16

Cite this article as: Hosokawa et al:: Observations of GPS scintillation during an isolated auroral substorm. Progress in Earth and Planetary Science 2014 1:16.

\section{Submit your manuscript to a SpringerOpen ${ }^{\circ}$ journal and benefit from:}

- Convenient online submission

- Rigorous peer review

- Immediate publication on acceptance

- Open access: articles freely available online

- High visibility within the field

- Retaining the copyright to your article

Submit your next manuscript at springeropen.com 